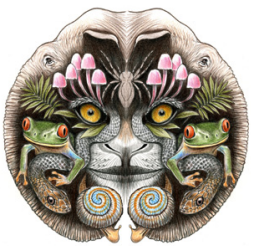

ISSN

Online 0974-7907

Print 0974-7893

OPEN ACCESS

\title{
Butterfles of Sundarban Biosphere Reserve, West Bengal, EASTERN INDIA: A PRELIMINARY SURVEY OF THEIR TAXONOMIC DIVERSITY, ECOLOGY AND THEIR CONSERVATION
}

\section{Soumyajit Chowdhury ${ }^{1,2}$}

\author{
${ }^{1}$ Centre for Biodiversity and Ecological Studies, P1/1B, Garia Park, Kolkata, West Bengal 700084, India; \\ ${ }^{2}$ Department of Zoology, V.J.R. College (affiliated to the University of Calcutta), 8/2, Bejoygarh, Jadavpur, Kolkata, \\ West Bengal 700032, India \\ wildlifesc@gmail.com
}

\begin{abstract}
The Indian Sundarbans, part of the globally famous deltaic eco-region, is little-studied for butterfly diversity and ecology. The present study reports 76 butterfly species belonging to five families, which is a culmination of 73 species obtained from surveys conducted over a period of three years (2009-2011) in reclaimed and mangrove forested areas and three species obtained from an earlier report. Six of these species are legally protected under the Indian Wildlife (Protection) Act, 1972. Random surveys were employed for both the study areas, supplemented by systematic sampling in reclaimed areas. The reclaimed and forested areas differed largely in butterfly richness (Whittaker's measure of $\beta$ diversity $=0.55$ ). For sample-based rarefaction curves, butterfly genera showed a tendency to reach an asymptote sooner than the species. Numerous monospecific genera ( $77.19 \%$ of the taxa) resulted in a very gentle but non-linear positive slope for the species-genus ratio curve. A species-genus ratio of 1.33 indicated strong intra-generic competition for the butterflies of the Indian Sundarbans. Mangrove areas were species poor, with rare species like Euploea crameri, Colotis amata and Idea agamarshchana being recorded in the mangrove area; while Danaus genutia was found to be the most frequent butterfly. Butterfly abundance was very poor, with no endemic species and the majority ( $53.9 \%$ of the taxa; $n=41$ ) were found locally rare. The changing composition of butterflies in the once species-poor mangrove zone of the fragile Sundarbans may interfere with their normal ecosystem functioning.
\end{abstract}

Keywords: Asclepiadaceae, butterfly, Danaus genutia, India, Mangrove, reclaimed area, Sundarbans.

DOI: http://dx.doi.org/10.11609/JoTT.03787.6082-92 | ZooBank: urn:Isid:zoobank.org:pub:4F1E7FBB-0BEA-4A43-AED1-2372A5AF57D8

Editor: B.A. Daniel, Zoo Outreach Organisation, Coimbatore, India.

Date of publication: 26 July 2014 (online \& print)

Manuscript details: Ms \# 03787 | Received 28 September 2013 | Final received 05 July 2014 | Finally accepted 08 July 2014

Citation: Chowdhury, S. (2014). Butterflies of Sundarban Biosphere Reserve, West Bengal, eastern India: a preliminary survey of their taxonomic diversity, ecology and their conservation. Journal of Threatened Taxa 6(8): 6082-6092; http://dx.doi.org/10.11609/JoTT.03787.6082-92

Copyright: @ Chowdhury 2014. Creative Commons Attribution 4.0 International License. JoTT allows unrestricted use of this article in any medium, reproduction and distribution by providing adequate credit to the authors and the source of publication.

Funding: Centre for Biodiversity and Ecological Studies [Ref No: CBES/2010/S-PI)]

Competing Interest: The author declares to have financial support from the Centre for Biodiversity and Ecological Studies (CBES) for the submitted work, and no other relationships or activities that have inspired the same.

Author Details: DR. SOUMYAIT CHOWDHURY is a faculty member of the Department of Zoology, V.J.R. College, Kolkata and founder member of Centre for Biodiversity and Ecological Studies (CBES). He has been working on the biodiversity and ecology of Indian insects and authored several research articles. He also initiated 'BIOME india', an online resource on the ecosystem-based conservation programmes of India.

Acknowledgement: The author is indebted to the Centre for Biodiversity and Ecological Studies for supporting, in part, the survey work on lepidopteran fauna and different mangrove and mangrove-associated floral components in Indian Sundarbans. Nihar Mondal, Tanmoy Mondal and Amit Das among several others deserve special thanks for logistic support during field trips in different parts of central, western and eastern Sundarbans.

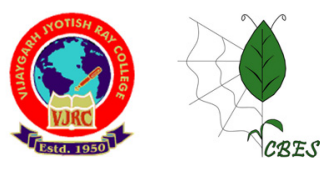




\section{INTRODUCTION}

Sundarbans forms a unique eco-region in the vast deltaic region on the Bay of Bengal, hosting one of the most extensive mangrove forests of the world, spreading over India and Bangladesh. This region is also the only mangrove tiger land in the world, securing the endangered Royal Bengal Tiger Panthera tigris tigris populations with inimitable cases of tigerhuman conflict. The human populations in this fragile eco-region have sustained themselves by harvesting forest products and by reclaiming the forest land for agricultural purposes, over the last two centuries. The usual flushing of tidal water and natural inundation has been greatly affected by embankment constructions in the Sundarbans, resulting in quick succession or shifting in plant communities (Sanyal et al. 1984).

Butterflies are recognized as focal species of conservation in several areas of the world (New 2011). These widely studied insects show significant ecological contributions in different ecosystems through herbivory and pollination services. The Indian Sundarbans, being a unique deltaic region with a rich biorepository, suffered from inadvertent reports on butterflies, accordingly lacking a detailed survey for the same. Literature reviews indicated a meagre eight species (Mandal \& Nandi 1989; Mandal \& Maulik 1997; Kehimkar 2008) to be precisely reported from this region. Hence, an inventory on the butterfly fauna in Indian Sundarbans and their mode of habitat association was required for this globally important eco-region.

\section{MATERIALS AND METHODS}

\section{Study Site}

The Indian Sundarbans, comprising an area of $9,630 \mathrm{~km}^{2}$ is part of the delta of the Ganga-BrahmaputraMeghna basin in Asia; and along with the Bangladesh Sundarbans hosts one of the largest continuous mangrove forests in the world. The landscape is characterized by a web of tidal water systems. Only the Hugli and Ichamati-Raimangal rivers carry freshwater flow of some significance (Danda et al. 2011). The Indian Sundarbans are located between $21^{\circ} 31^{\prime}-22^{\circ} 30^{\prime} \mathrm{N}$ and $88^{\circ} 10^{\prime}-89^{\circ} 51^{\prime} \mathrm{E}$, spreading over the southern parts in the districts of North and South 24-Parganas of the state of West Bengal (Naskar \& Mandal 1999) and demarcated by the 'Dampier and Hodges Line' in the north. This area has received the status of Sundarbans Biosphere Reserve (SBR) in 1989, by being the world's first mangrove forest to be brought under scientific management. Mangrove forests cover $4,264 \mathrm{~km}^{2}$ and include a tiger reserve covering $2,585 \mathrm{~km}^{2}$, a national park covering $1,330 \mathrm{~km}^{2}$ and three wildlife sanctuaries, namely Sajnekhali WS $\left(362 \mathrm{~km}^{2}\right)$, Lothian Island WS $\left(39 \mathrm{~km}^{2}\right)$ and Halliday Island WLS $\left(5.8 \mathrm{~km}^{2}\right)$. The remaining area, covering $5,366 \mathrm{~km}^{2}$, comprises reclaimed land (Chaudhuri \& Choudhury 1994; Zockler et al. 2005).

The region experiences a humid, tropical, maritime climate, with an average annual rainfall of $142.5 \mathrm{~mm}$. Mean annual maximum and minimum temperatures are about $30^{\circ} \mathrm{C}$ and $23^{\circ} \mathrm{C}$ respectively. Humidity is high, $80 \%$ on average due to proximity to the Bay of Bengal (Chowdhury 2011). Recent reports suggest that air temperature over the Sundarbans and adjacent parts of Bay of Bengal are gradually increasing (Huq et al. 1999; Agrawala et al. 2003).

Based on the vegetation pattern and extent of exposure to saline water regimes, two broad categories of eco-zones were recognized among the 18 sites that were sampled in the eastern, central and western Sundarbans (Fig. 1): 1 - Mangrove area and 2 - Reclaimed area. The former is exposed to saline tidal submergence with luxuriant mangrove vegetation (Image 1), while the latter is retained for human settlements and agricultural practices with low saline influence and non-mangrove vegetation (Image 2).

\section{Butterfly sampling and data analyses}

Butterflies were sampled by visual estimation surveys for three years: 2009-2011; with samplings repeated for pre-monsoon, monsoon and post-monsoon months of the study period. Sampling of butterflies varied according to habitat patterns over the deltaic region. In the mangrove forested regions, butterflies were broadly sampled in a random manner along the forest edges and trails available. Whereas in the reclaimed areas, surveys were carried out especially in the greener areas (viz., gardens, agricultural fields, fragmented wooded areas) so as to encounter butterflies more frequently as compared to the highly degraded and modified locales. Both random searches and systematic sampling (along definite trails) were carried out in the latter. Butterflies were recorded from 0800-1700 hr each day. Species were observed while perched on surrounding vegetation, in flight and puddling as well as nectar feeding were recorded and photographed in situ for reference. Species identifications were done following available literature (Evans 1932; Wynter-Blyth 1957; Kehimkar 2008). Taxonomic classification of butterfly families was followed after van Nieukerken et al. (2011). 


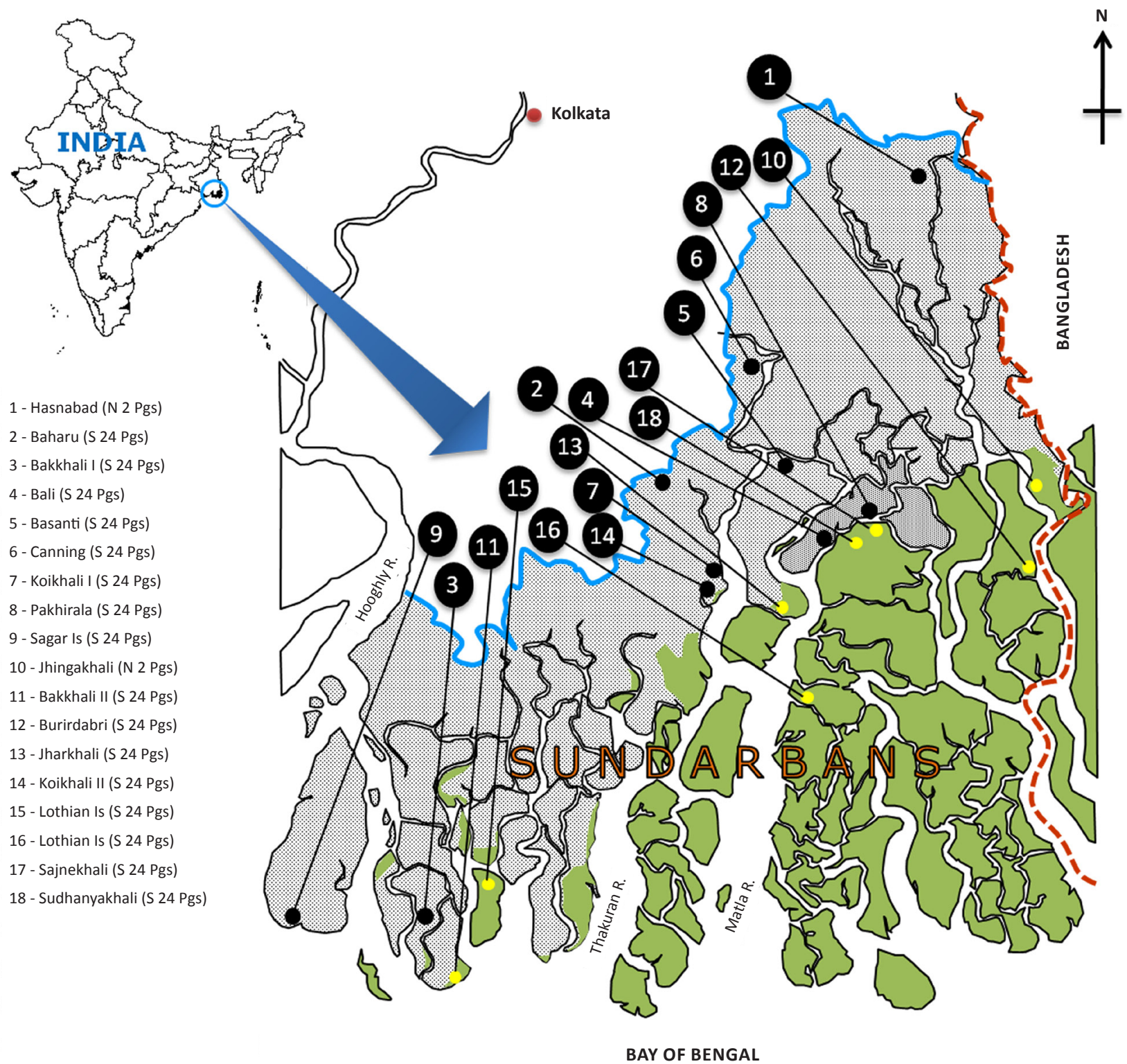

Mangrove Forest Recliamed Area
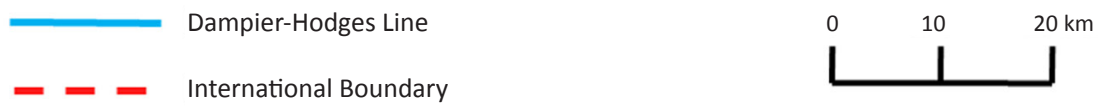

Figure 1. A map of the Indian Sundarbans delta, showing the sampling sites encompassing the North and South 24 Parganas districts of the state of West Bengal, India.

The presence-absence data for butterflies in the sampling sites in the western, central, and eastern Sundarbans were pooled to form a species $X$ sample incidence matrix. Taxon sampling curves (samplebased rarefaction curves) for butterfly species and for the genera to which they belong were generated using
Estimate S version 9.1.0 (Colwell 2013); their speciesto-genus ratios were plotted along with so as to deduce levels of competitive interactions among species within genera (Simberloff 1978; Järvinen 1982).

Estimation of $\beta$-diversity (diversity between habitats) between the mangrove forests and reclaimed 


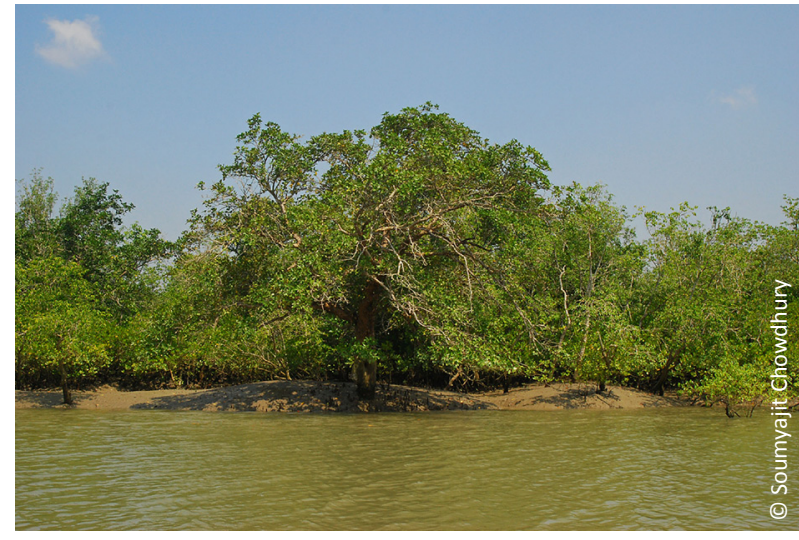

Image 1. Mangrove forest at Sudhanyakhali in Sundarbans, India.

areas of the Sundarban deltaic region were done using Whittaker's measure $\beta_{w}$ (Whittaker 1960) for presence/ absence data (as in the present case):

$$
\beta_{w}=S / \alpha \text {. }
$$

where $S=$ the total number of species recorded in both sites, and $\alpha=$ the average sample richness. The obtained result was calculated on the 0 (minimum $\beta$ diversity) to 1 (maximum $\beta$ diversity) scale by subtracting 1 from the obtained answer (Magurran 2004).

Frequency of occurrence (FO) of the butterfly species was calculated by considering the number of sampled sites in which the species occurred in relation to the total number of samples (or sites) surveyed (Severiano et al. 2012). Such an analysis was viewed into the following categories when FO > 70\% [very frequent (VF)]; $\leq 70 \%$ $->45 \%$ [frequent (F); $\leq 45 \%->15 \%$ [Infrequent (IF)]; $\leq$ $15 \%$ [Rare].

\section{RESULTS}

A total of 76 species of butterflies have been reported hitherto from Indian Sundarbans (Table 1). Of these, 73 species were recorded following the three years (2009-2011) field survey. The remaining three species, viz., Graphium nomius, Eurema brigitta and Idea agamarschana, were recorded by Mandal \& Nandi (1989) in Sundarbans but not recorded in the present survey. All the following analyses have been done with respect to the 76 species.

Among the five recorded butterfly families in Sundarbans, Nymphalidae appears to be most species rich $(n=24,31.6 \%)$, followed by Lycaenidae $(n=19,25 \%)$. The family with lowest species richness is Papilionidae $(n=9,11.8 \%)$. Pieridae and Hesperiidae were intermediate with $17.1 \%(n=13)$ and $14.5 \%(n=11)$ of the

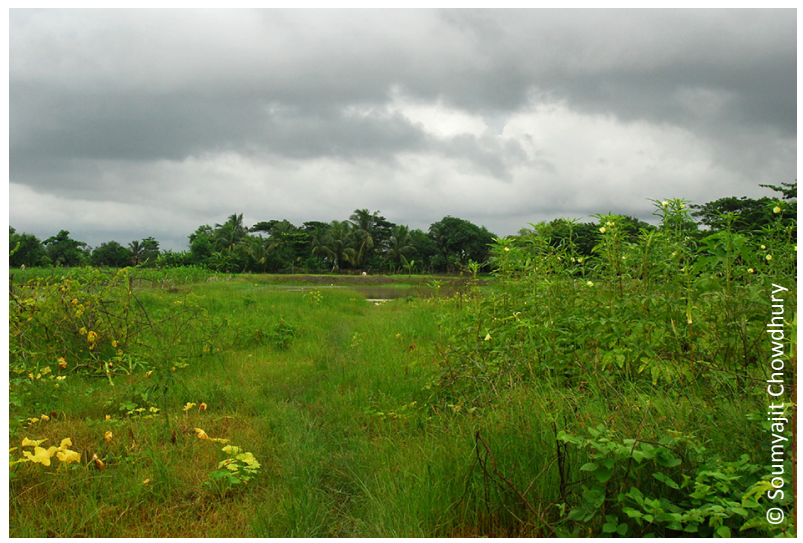

Image 2. Reclaimed area at Basanti in Sundarbans, India.

total species. Regarding genus richness, the butterfly families followed nearly the same trend as their species; nymphalids ( $n=16,28 \%$ ) outnumber the others while papilionids ranked last $(n=3,5.2 \%)$ in the list (Table 2$)$.

The distribution of butterfly species across genera was found to be highly skewed. A large proportion of genera (44 out of 57) recorded in the present study were represented by single species, whereas the remaining eight genera by two species, four genera by three species and one genus by four species (Fig. 2). Figure 3 illustrates the most prevalent genera, that is, those with three and four species.

Figure 4 depicts sample-based species and genus rarefaction curves for the butterfly dataset. Both the curves do not reach a plateau with increasing sample numbers. However, the number of genera shows a tendency to reach an asymptote sooner than the number of species. Moreover, a very low speciesgenus ratio ( $\mathrm{S} / \mathrm{G}=1.33$ ) exists for the butterflies of Indian Sundarbans delta.

A fairly high beta diversity for the butterfly fauna was indicated by the obtained value $\left(\beta_{w}=0.55\right)$ of the Whittaker's measure between the mangrove forests and reclaimed areas of Indian Sundarbans. All the butterfly families show a higher species richness in the reclaimed areas than in the mangrove forest zones (Fig. 5). Whereas Nymphalidae, Lycaenidae and Hesperiidae fall away by about $50 \%$ in mangroves as compared to reclaimed areas, a marked drop can be seen in Lycaenidae and Hesperiidae (by < 20\%) in mangrove forests as against reclaimed areas.

Of the 76 species of butterflies, most are 'common' and 'generalist' as none of the species are threatened globally as per the IUCN Red List (2011). However, regarding their frequency of occurrence in the Sundarbans, the majority of butterflies were classified as 
Table 1. A species list of the butterfly fauna in Indian Sundarbans. The preferential occurrences of the species are indicated by specific ecozones in which they were recorded, being abbreviated as MF: mangrove forest and RA: reclaimed (non-forest) area

\begin{tabular}{|c|c|c|c|}
\hline & Taxa & Occurrence & FO \\
\hline \multicolumn{4}{|c|}{ Superfamily Papilionoidea } \\
\hline \multicolumn{4}{|c|}{ Family Papilionidae } \\
\hline \multicolumn{4}{|c|}{ Subfamily Papilioninae } \\
\hline 1 & $\begin{array}{l}\text { Common Jay Graphium doson (C \& R } \\
\text { Felder, 1864) }\end{array}$ & RA & IF \\
\hline 2 & $\begin{array}{l}\text { Tailed Jay Graphium agamemnon } \\
\text { (Linnaeus, 1758) }\end{array}$ & RA & IF \\
\hline 3 & Lime Papilio demoleus (Linnaeus, 1758) & $\mathrm{RA}, \mathrm{MF}$ & $\mathrm{IF}$ \\
\hline 4 & $\begin{array}{l}\text { Common Mime Papilio clytia (Linnaeus, } \\
\text { 1758) }\end{array}$ & $\mathrm{RA}, \mathrm{MF}$ & $\mathrm{R}$ \\
\hline 5 & $\begin{array}{l}\text { Blue Mormon Papilio polymnestor (Cramer, } \\
\text { 1775) }\end{array}$ & RA & $\mathrm{R}$ \\
\hline 6 & $\begin{array}{l}\text { Common Mormon Papilio polytes } \\
\text { (Linnaeus, 1758) }\end{array}$ & RA, MF & $\mathrm{F}$ \\
\hline 7 & $\begin{array}{l}\text { Common Rose Pachliopta aristolochiae } \\
\text { (Fabricius, 1775) }\end{array}$ & RA, MF & IF \\
\hline 8 & $\begin{array}{l}\text { Crimson Rose Pachliopta hector (Linnaeus, } \\
1758)^{+}\end{array}$ & RA, MF & IF \\
\hline 9 & $\begin{array}{l}\text { Spot Swordtail Graphium nomius (Esper, } \\
1798)^{*}\end{array}$ & MF & $\mathrm{R}$ \\
\hline \multicolumn{4}{|c|}{ Family Hesperiidae } \\
\hline \multicolumn{4}{|c|}{ Subfamily Coeliadinae } \\
\hline 10 & $\begin{array}{l}\text { Brown Awl Badamia exclamationis } \\
\text { (Fabricius, 1775) }\end{array}$ & RA & $\mathrm{R}$ \\
\hline \multicolumn{4}{|c|}{ Subfamily: Hesperiinae } \\
\hline 11 & $\begin{array}{l}\text { Chestnut Bob lambrix salsala (Moore, } \\
\text { 1866) }\end{array}$ & RA & $\mathrm{R}$ \\
\hline 12 & $\begin{array}{l}\text { Indian Bob Palm Suastus gremius } \\
\text { (Fabricius, 1798) }\end{array}$ & RA & $\mathrm{R}$ \\
\hline 13 & $\begin{array}{l}\text { Dark Palm Dart Telicota ancilla (Herrich- } \\
\text { Schäffer, 1869) }\end{array}$ & RA & $\mathrm{R}$ \\
\hline 14 & $\begin{array}{l}\text { Common Dartlet Oriens gola (Moore, } \\
\text { 1877) }\end{array}$ & RA & $\mathrm{R}$ \\
\hline 15 & Grass Demon Udaspes folus (Cramer, 1775) & RA & $\mathrm{R}$ \\
\hline 16 & $\begin{array}{l}\text { Common Red Eye Matapa aria (Moore, } \\
\text { 1866) }\end{array}$ & RA & $\mathrm{R}$ \\
\hline 17 & $\begin{array}{l}\text { Common Straight Swift Parnara guttata } \\
\text { (Bremer and Grey, 1852) }\end{array}$ & RA, MF & IF \\
\hline 18 & Rice Swift Borbo cinnara (Wallace, 1866) & RA & $\mathrm{IF}$ \\
\hline 19 & $\begin{array}{l}\text { Small Branded Swift Pelopidas mathias } \\
\text { (Fabricius, 1798) }\end{array}$ & RA, MF & IF \\
\hline \multicolumn{4}{|c|}{ Subfamily Pyrginae } \\
\hline 20 & $\begin{array}{l}\text { Common Snow Flat Tagiades japetus (Stoll, } \\
\text { 1781) }\end{array}$ & RA & $\mathrm{R}$ \\
\hline \multicolumn{4}{|c|}{ Family Pieridae } \\
\hline \multicolumn{4}{|c|}{ Subfamily Coliadinae } \\
\hline 21 & 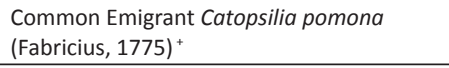 & RA & $\mathrm{R}$ \\
\hline 22 & $\begin{array}{l}\text { Mottled Emigrant Catopsilia pyranthe } \\
\text { (Linnaeus, 1758) }\end{array}$ & RA, MF & $\mathrm{F}$ \\
\hline 23 & $\begin{array}{l}\text { Common Grass Yellow Eurema hecabe } \\
\text { (Linnaeus, 1758) }\end{array}$ & RA, MF & $\mathrm{F}$ \\
\hline 24 & $\begin{array}{l}\text { Small Grass Yellow Eurema brigitta } \\
\text { (Cramer, 1780) * }\end{array}$ & MF & $\mathrm{R}$ \\
\hline 25 & $\begin{array}{l}\text { Three-spot Grass Yellow Eurema blanda } \\
\text { (Boisduval, 1836) }\end{array}$ & RA & $\mathrm{R}$ \\
\hline
\end{tabular}

\begin{tabular}{|c|c|c|c|}
\hline & Taxa & Occurrence & FO \\
\hline \multicolumn{4}{|c|}{ Subfamily Pierinae } \\
\hline 26 & $\begin{array}{l}\text { Common Albatross Appias albina } \\
\text { (Boisduval, 1836) }\end{array}$ & RA & $\mathrm{R}$ \\
\hline 27 & $\begin{array}{l}\text { Striped Albatross Appias libythea } \\
\text { (Fabricius, 1775) }\end{array}$ & RA, MF & $\mathrm{IF}$ \\
\hline 28 & $\begin{array}{l}\text { Small Salmon Arab Colotis amata } \\
\text { (Fabricius, 1775) }\end{array}$ & RA & $\mathrm{R}$ \\
\hline 29 & $\begin{array}{l}\text { Common Gull Cepora nerissa (Fabricius, } \\
\text { 1775) }\end{array}$ & RA, MF & $\mathrm{R}$ \\
\hline 30 & $\begin{array}{l}\text { Common Jezebel Delias eucharis (Drury, } \\
\text { 1773) }\end{array}$ & RA, MF & IF \\
\hline 31 & $\begin{array}{l}\text { Yellow Orange Tip Ixias pyrene (Linnaeus, } \\
\text { 1764) }\end{array}$ & RA & $\mathrm{R}$ \\
\hline 32 & Psyche Leptosia nina (Fabricius, 1793) & RA & IF \\
\hline 33 & $\begin{array}{l}\text { Common Wanderer Pareronia valeria } \\
\text { (Cramer, 1776) }\end{array}$ & RA & $\mathrm{R}$ \\
\hline \multicolumn{4}{|c|}{ Family Lycaenidae } \\
\hline \multicolumn{4}{|c|}{ Subfamily Curetinae } \\
\hline 34 & $\begin{array}{l}\text { Indian Sunbeam Curetis thetis (Drury, } \\
1773 \text { ) }\end{array}$ & RA & $\mathrm{R}$ \\
\hline \multicolumn{4}{|c|}{ Subfamily Polyommatinae } \\
\hline 35 & $\begin{array}{l}\text { Gram Blue Euchrysops cnejus (Fabricius, } \\
\text { 1798) }\end{array}$ & RA & IF \\
\hline 36 & Lime Blue Chilades laius (Stoll, 1780) & RA & $\mathrm{R}$ \\
\hline 37 & $\begin{array}{l}\text { Pea Blue Lampides boeticus (Linnaeus, } \\
\text { 1767) }\end{array}$ & RA & IF \\
\hline 38 & $\begin{array}{l}\text { Common Cerulean Jamides celeno } \\
\text { (Cramer, 1775) }\end{array}$ & RA & $\mathrm{R}$ \\
\hline 39 & $\begin{array}{l}\text { Forget-me-not Catochrysops strabo } \\
\text { (Fabricius, 1793) }\end{array}$ & RA & $\mathrm{R}$ \\
\hline 40 & $\begin{array}{l}\text { Dark Grass Blue Zizeeria karsandra } \\
\text { (Moore, 1865) }\end{array}$ & RA & IF \\
\hline 41 & $\begin{array}{l}\text { Lesser Grass Blue Zizena otis (Fabricius, } \\
\text { 1787) }\end{array}$ & RA & $\mathrm{R}$ \\
\hline 42 & $\begin{array}{l}\text { Tiny Grass Blue Zizula hylax (Fabricius, } \\
\text { 1775) }\end{array}$ & RA & IF \\
\hline 43 & $\begin{array}{l}\text { Common Lineblue Prosotas nora (C \& R } \\
\text { Felder, 1860) }\end{array}$ & MF & $\mathrm{R}$ \\
\hline 44 & $\begin{array}{l}\text { Common Pierrot Castalius rosimon } \\
\text { (Fabricius, 1775) }\end{array}$ & RA & $\mathrm{R}$ \\
\hline 45 & $\begin{array}{l}\text { Rounded Pierrot Tarucus nara (Kollar, } \\
\text { 1848) }\end{array}$ & RA & $\mathrm{R}$ \\
\hline 46 & $\begin{array}{l}\text { Quaker Neopithecops zalmora (Butler, } \\
\text { 1870) }\end{array}$ & RA & $\mathrm{IF}$ \\
\hline \multicolumn{4}{|c|}{ Subfamily Theclinae } \\
\hline 47 & $\begin{array}{l}\text { Common Red Flash Rapala iarbus } \\
\text { (Fabricius, 1787) }\end{array}$ & RA & $\mathrm{R}$ \\
\hline 48 & $\begin{array}{l}\text { Slate Flash Rapala manea (Hewitson, } \\
\text { 1863) }\end{array}$ & RA & $\mathrm{R}$ \\
\hline 49 & $\begin{array}{l}\text { Monkey Puzzle Rathinda amor (Fabricius, } \\
\text { 1775) }\end{array}$ & RA & R \\
\hline 50 & $\begin{array}{l}\text { Falcate Oak Blue Mahathala ameria } \\
\text { (Hewitson, 1832) }\end{array}$ & RA & $\mathrm{R}$ \\
\hline 51 & $\begin{array}{l}\text { Common Silverline Spindasis vulcanus } \\
\text { (Fabricius, 1775) }\end{array}$ & RA & $\mathrm{R}$ \\
\hline 52 & Yamfly Loxura atymnus (Stoll, 1780) & RA & $\mathrm{R}$ \\
\hline
\end{tabular}




\begin{tabular}{|c|c|c|c|}
\hline & Taxa & Occurrence & FO \\
\hline \multicolumn{4}{|c|}{ Family Nymphalidae } \\
\hline \multicolumn{4}{|c|}{ Subfamily Biblidinae } \\
\hline 53 & $\begin{array}{l}\text { Angled Castor Ariadne ariadne (Linnaeus, } \\
\text { 1758) }\end{array}$ & RA & $\mathrm{R}$ \\
\hline 54 & $\begin{array}{l}\text { Common Castor Ariadne merione (Cramer, } \\
\text { 1779) }\end{array}$ & RA & IF \\
\hline \multicolumn{4}{|c|}{ Subfamily Danainae } \\
\hline 55 & $\begin{array}{l}\text { Common Indian Crow Euploea core } \\
\text { (Cramer, 1780) }\end{array}$ & RA, MF & $\mathrm{F}$ \\
\hline 56 & $\begin{array}{l}\text { Spotted Black Crow Euploea crameri } \\
\text { (Lucas, 1853) }^{+}\end{array}$ & RA, MF & $\mathrm{R}$ \\
\hline 57 & $\begin{array}{l}\text { Blue Tiger Tirumala limniace (Cramer, } \\
\text { 1775) }\end{array}$ & RA, MF & $\mathrm{F}$ \\
\hline 58 & $\begin{array}{l}\text { Plain Tiger Danaus chrysippus (Linnaeus, } \\
\text { 1758) }\end{array}$ & RA, MF & $\mathrm{F}$ \\
\hline 59 & $\begin{array}{l}\text { Striped Tiger Danaus genutia (Cramer, } \\
1779)^{+}\end{array}$ & RA, MF & $\mathrm{F}$ \\
\hline 60 & $\begin{array}{l}\text { White Tiger Danaus melanippus (Cramer, } \\
\text { 1777) }{ }^{+}\end{array}$ & RA, MF & IF \\
\hline 61 & $\begin{array}{l}\text { Tree Nymph Idea agamarshchana (C and R } \\
\text { Felder, 1865) }{ }^{+}\end{array}$ & MF & $\mathrm{R}$ \\
\hline \multicolumn{4}{|c|}{ Subamily Heliconiinae } \\
\hline 62 & $\begin{array}{l}\text { Tawny Coster Acraea violae (Fabricius, } \\
\text { 1775) }\end{array}$ & RA & IF \\
\hline 63 & $\begin{array}{l}\text { Common Leopard Phalanta phalantha } \\
\text { (Drury, 1773) }\end{array}$ & RA & IF \\
\hline \multicolumn{4}{|c|}{ Subfamily Limenitinae } \\
\hline 64 & $\begin{array}{l}\text { Common Baron Euthalea aconthea } \\
\text { (Cramer, 1777) }\end{array}$ & RA & IF \\
\hline 65 & $\begin{array}{l}\text { Commander Moduza procris (Cramer, } \\
\text { 1777) }\end{array}$ & RA & IF \\
\hline 66 & Common Sailer Neptis hylas (Moore, 1758) & RA & $\mathrm{R}$ \\
\hline \multicolumn{4}{|c|}{ Subfamily Nymphalinae } \\
\hline 67 & $\begin{array}{l}\text { Great Eggfly Hypolimnas bolina (Linnaeus, } \\
\text { 1758) }\end{array}$ & RA, MF & IF \\
\hline 68 & Grey Pansy Junonia atlites (Linnaeus, 1763) & RA, MF & IF \\
\hline 69 & $\begin{array}{l}\text { Lemon Pansy Junonia lemonias (Linnaeus, } \\
\text { 1758) }\end{array}$ & RA & $\mathrm{R}$ \\
\hline 70 & $\begin{array}{l}\text { Peacock Pansy Junonia almana (Linnaeus, } \\
\text { 1758) }\end{array}$ & RA & IF \\
\hline \multicolumn{4}{|c|}{ Subfamily Satyrinae } \\
\hline 71 & $\begin{array}{l}\text { Common Bushbrown Mycalesis perseus } \\
\text { (Fabricius, 1775) }\end{array}$ & RA, MF & IF \\
\hline 72 & $\begin{array}{l}\text { Dark-Brand Bushbrown Mycalesis mineus } \\
\text { (Linnaeus, 1758) }\end{array}$ & RA & $\mathrm{R}$ \\
\hline 73 & $\begin{array}{l}\text { Common Evening Brown Melanitis leda } \\
\text { (Linnaeus, 1758) }\end{array}$ & RA & IF \\
\hline 74 & $\begin{array}{l}\text { Common Palmfly Elymnias hypermenstra } \\
\text { (Linnaeus, 1758) }\end{array}$ & RA, MF & $\mathrm{F}$ \\
\hline 75 & $\begin{array}{l}\text { Common Five Ring Ypthima baldus } \\
\text { (Fabricius, 1775) }\end{array}$ & RA & $\mathrm{R}$ \\
\hline 76 & $\begin{array}{l}\text { Common Four Ring Ypthima huebneri } \\
\text { (Kirby, 1871) }\end{array}$ & RA & $\mathrm{R}$ \\
\hline
\end{tabular}

$\mathrm{FO}=$ Frequency of Occurrence, $\mathrm{VF}=$ Very Frequent, $\mathrm{F}=$ Frequent, $\mathrm{I}=$ Infrequent $\mathrm{R}=$ Rare

* Species reported in Mandal and Nandi (1989)

${ }^{+}$Species reported from both literature reviews and field surveys

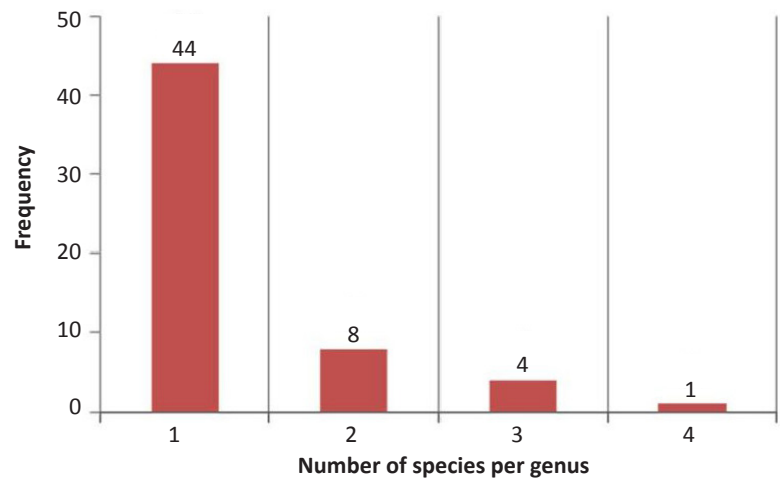

Figure 2. Frequency of the number of butterfly species recorded for each genus reported from Indian Sundarbans. The total number of observation in each category is shown on top of the bar. Most of the genera were represented by single species.

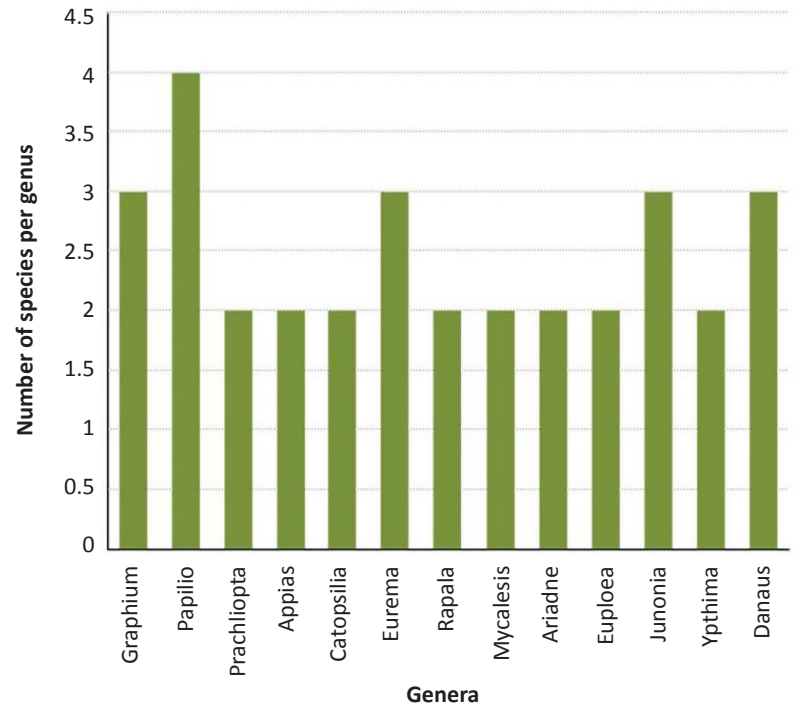

Figure 3. Most species-rich genera of butterflies of Sundarbans, comprising of two or more species hitherto recorded from this region.

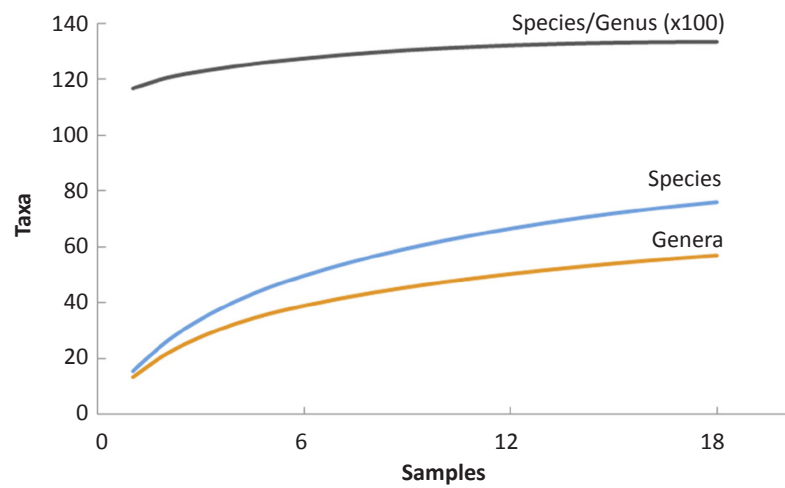

Figure 4. Taxon sampling curves for species and for the genera of butterfly fauna of Indian Sundarbans delta to which they belong, with the species-genus ratio. 
Table 2. Overview of the taxonomic diversity of butterflies of Sundarbans.

\begin{tabular}{|l|l|l|l|}
\hline Family & Subfamily & Genera & Species \\
\hline Papilionidae & $1(6.7 \%)$ & $3(5.2 \%)$ & $9(11.8 \%)$ \\
\hline Hesperiidae & $3(20 \%)$ & $11(19.3 \%)$ & $11(14.5 \%)$ \\
\hline Pieridae & $2(13.3 \%)$ & $9(15.7 \%)$ & $13(17.1 \%)$ \\
\hline Lycaenidae & $3(20 \%)$ & $18(31.7 \%)$ & $19(25.0 \%)$ \\
\hline Nymphalidae & $6(40 \%)$ & $16(28.1 \%)$ & $24(31.6 \%)$ \\
\hline Total: 5 & $15(100 \%)$ & $57(100 \%)$ & $76(100 \%)$ \\
\hline
\end{tabular}

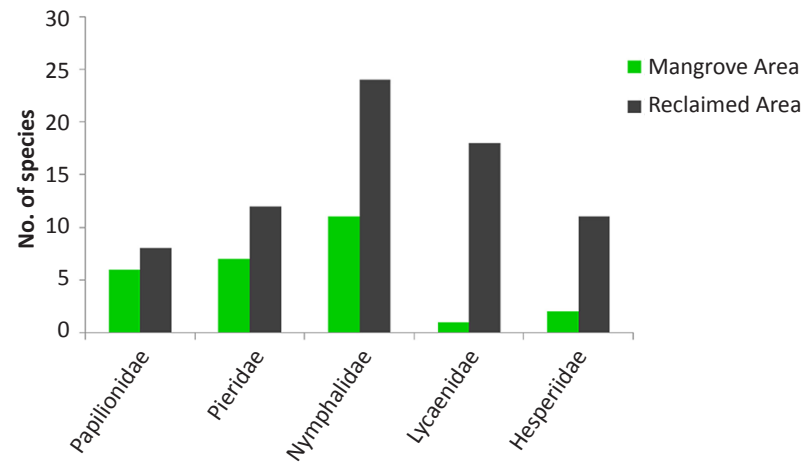

Figure 5. Butterfly families compared among mangrove and reclaimed areas in Indian Sundarbans in terms of their species richness.

'rare' ( $n=41 ; 53.9 \%$ of the taxa), as they were recorded only in one or two sites. Most of the rare species belonged to Lycaenidae and Hesperiidae (Fig. 6).

No butterfly species in the Sundarbans were recorded as 'very frequent' during the study period. A meagre $10.5 \%$ of the species $(n=8)$ were found to be 'frequent' in both the reclaimed areas and the mangrove forests (Fig. 6). Hesperiidae and Lycaenidae families remained devoid of any frequently observed species (Fig. 6). Three species of Tiger butterflies, viz., Danaus genutia Cramer, D. chrysippus Linnaeus and Tirumala limniace Cramer were recorded with FO>60\%. Striped Tiger, Danaus genutia was the only species to occur in most of the sampled sites (12 out of 18 sites). Besides this, the deltaic region does not hold any endemic species of butterflies.

Of the 76 butterfly species in the Indian Sundarbans, six are legally protected under the Indian Wildlife (Protection) Act, 1972 (Table 3).
Table 3. Legally protected butterflies of Sundarbans under the Indian Wildlife (Protection) Act, 1972 (WPA).

\begin{tabular}{|l|l|l|}
\hline WPA Schedule & Butterfly family & Species \\
\hline \multirow{2}{*}{ Schedule I } & Papilionidae & Pachliopta hector (Crimson Rose) \\
\cline { 2 - 3 } & Nymphalidae & Euploea crameri (Spotted Black Crow) \\
\hline Total Number of protected species under Schedule I of WPA: 2 \\
\hline \multirow{2}{*}{ Schedule II } & Lycaenidae & $\begin{array}{l}\text { Euchrysops cnejus (Gram Blue) } \\
\text { Lampides boeticus (Pea Blue) } \\
\text { Mahathala ameria (Falcate Oak Blue) }\end{array}$ \\
\hline Total Number of protected species under Schedule II of WPA: 3 \\
\hline Schedule IV & Pieridae & Appias libythea (Striped Albatross) \\
\hline \multicolumn{2}{|l|}{ Total Number of protected species under Schedule IV of WPA: 1 } \\
\hline
\end{tabular}

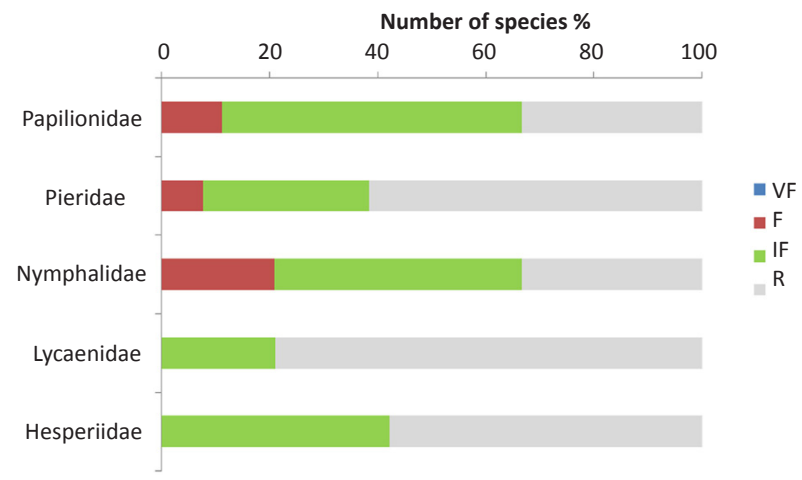

Figure 6. The categories of frequency of occurrence (FO) for butterfly families in Indian Sundarbans. VF = Very Frequent; F = Frequent; $\mathrm{IF}=$ Infrequent; $\mathrm{R}=$ Rare. Typical features include absence of 'Very Frequent' class and presence of 'Rare' class in maximum, with lycaenids and hesperiids taking the greatest share.

\section{DISCUSSION}

As compared to an inventory of 73 butterfly species from the Indian Sundarbans during the present study, earlier workers like Mandal \& Nandi (1989) recorded eight species only. Species sampled during the field surveys when compared to that obtained from literature surveys revealed that three species, viz., Graphium nomius, Eurema brigitta and Idea agamarschana were not recorded by the author during his present study. These three butterflies were reported to occur in both, the dense and the scanty mangrove forests of the Indian Sundarbans (Mandal \& Nandi 1989). The present article thus includes these three species (marked with* in Table 1) along with the species inventoried to obtain a total of 76 butterfly species in the Indian Sundarbans (Table 1).

Butterfly species recorded in the Indian Sundarbans were found to be distributed among five families: Papilionidae, Hesperiidae, Pieridae, Lycaenidae and 
Nymphalidae, under a single superfamily Papilionoidea (van Nieukerken et al. 2011). Large variations have been found to exist among the butterflies at both their generic and specific categories as reflected in Table 2 . Such variations can be attributed to the habitat heterogeneity and diversity of the range of larval host plants that have colonized the delta from the mainland over the years, primarily due to reclaiming of the previously occurring dense mangrove forest lands for colonization, agriculture and aquaculture practices.

The tendency of the sample-based species and genus rarefaction curves, not to reach a plateau with increasing sample numbers, indicates an addition of some newer species with further sampling. However, the tendency of the number of genera to reach an asymptote sooner than the number of species sampled reflects a pattern inevitable for any case of category-subcategory sampling curves (Gotelli \& Colwell 2001). Although the genus has fewer members than the species in the present case, the respective rarefaction curves are closely placed because of the occurrence of numerous monospecific genera ( $n=44 ; 77.19 \%$ of the taxa) of butterflies in the Indian Sundarbans. This unique relationship is reflected in the species-genus ratio curve (Fig. 4) revealing a very gentle but non-linear positive slope.

The species-genus ratio has long been in practice to describe community patterns as well as to surmise levels of competitive interactions among species within genera (Gotelli \& Colwell 2001). A very low species-genus ratio $(S / G=1.33)$ for the butterflies of the Sundarbans, following Elton's (1946) proposition, indicates strong intra-generic competition, limiting in turn congeneric coexistence as hypothesized by Darwin (1859). Widespread observations revealed that species-genus ratios were usually smaller for island than mainland communities (Elton 1946; Simberloff 1978). As most of the Indian Sundarbans delta is comprised of island complexes, the present findings remain consistent with the aforementioned propositions.

Interference in intrusion of saline water (resulting from tidal influx) and reclamation of forest lands for the ever-growing demand for human settlement and agricultural practices in the upper and middle estuarine regions of the Sundarbans has resulted in modifying the floristic spectrum, indulging the intensification of a diverse array of larval food plants as well as nectar plants for adult butterflies. Such a phenomenon facilitated an immigration of diverse butterflies from inland areasthereby changing their community composition in the butterfly-poor mangrove ecosystems and the nonforest areas of the Indian Sundarbans delta. Deficient food sources, particularly in terms of non-nutritious mangrove leaves and stressed environmental conditions, like strong sunlight, high temperature and desiccation (Kathiresan \& Bingham 2001) may have made their species richness in such ecosystems correspondingly low. This is reflected by their fairly high beta diversity existing between reclaimed and mangrove forested areas of the Indian Sundarbans. Earlier studies also indicate fewer species to be restricted to mangroves in other parts of the world (Corbet \& Pendelbury 1992). Thus, many of the species recorded in the Sundarban mangrove forests in the present study can be suggested to be visitors that frequently visit the mangrove flowers for the rich nectar sources.

Although all butterfly families show a higher species richness in reclaimed areas, prominent contribution towards their difference among the reclaimed and mangrove areas were largely attributed to the lycaenids and hesperiids (Fig. 5). The underlying reasons for such an event seem to be twofold: (i) the extreme deficiency in larval food plants for these two families in the mangrove forests and (ii) the increased abundance of various cultivated and decorative plants (Arecaceae, Combretaceae, Fabaceae, Mimosaceae, Poaceae, Rhamnaceae, Rubiaceae, Rutaceae, Zinziberacaeae and others) in reclaimed areas of the Indian Sundarbans that serve as preferred food plants for their larvae.

Forty one species of butterflies in Sundarbans have been considered rare in terms of their frequency of occurrence in the deltaic region. Rare species like the Spotted Black Crow Euploea crameri Lucas is a resident of the mangrove belt (Mondal \& Maulik 1997), although it was mentioned to be accidentally introduced by shipping on a small, flat alluvial island of Sundarbans (Fruhstorfer 1910). Mangrove Nymph I. agamarshchana C. \& R. Felder is rare in the Indian Sundarbans, from

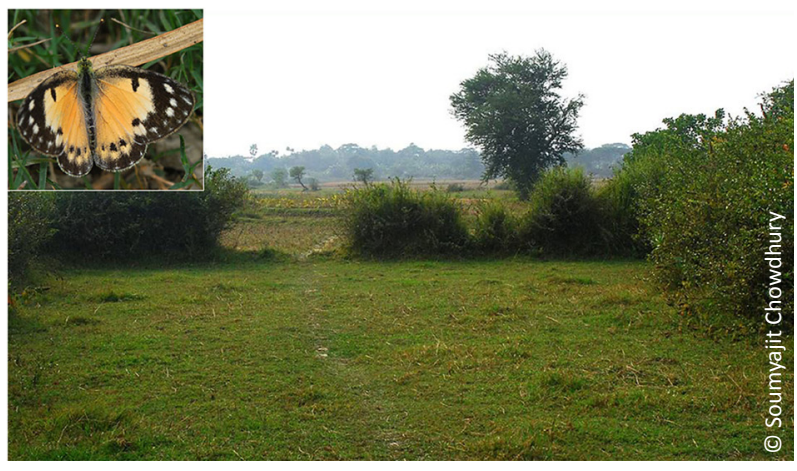

Image 3. The degrading habitat for Small Salmon Arab Colotis amata (Fabricius, 1775) at a reclaimed area in Canning, Indian Sundarbans. The inset shows a male individual of the species. 

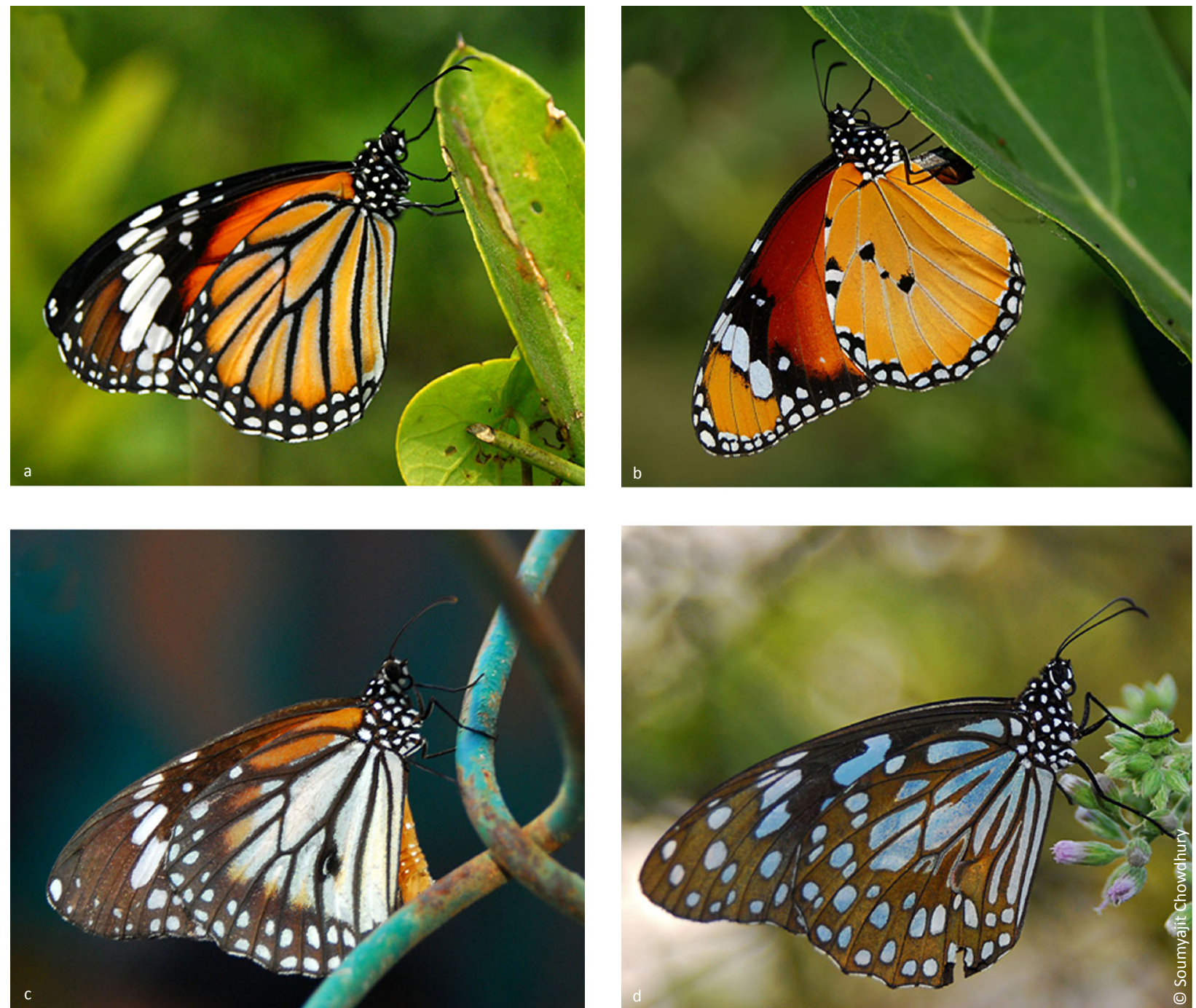

Image 4. Tiger Butterflies of Indian Sundarbans delta: (a) Striped Tiger Danaus genutia (Cramer, 1779), (b) Plain Tiger Danaus chrysippus (Linnaeus, 1758), (c) White Tiger Danaus melanippus (Cramer, 1777), (d) Blue Tiger Tirumala limniace (Cramer, 1775).

where it was first reported by Mondal \& Maulik (1997); although Talbot (1949) mentioned it hitherto as very common in the mangrove forests of the Bangladesh Sundarbans. Another rare butterfly-the Small Salmon Arab Colotis amata Fabricius, being a 'specialist' and monophagous on Azima tetracantha (a semi-saline, mangrove-associated species belonging to family Salvadoraceae) was found to be locally threatened because of habitat modifications and rapid depletion of the host plant populations (Chowdhury \& Soren 2009) in some reclaimed areas of the Sundarbans (Image 3 ).

The deltaic environment of the Sundarbans proved hostile for butterfly abundance as no butterfly species were found to be 'very frequent' (Fig. 6). Only 8 species were found to be 'frequent' in both the reclaimed areas and mangrove forests, viz., the papilionid Papilio polytes
Linnaeus, the pierids Catopsilia pyranthe Linnaeus and Eurema hecabe Linnaeus, and the nymphalids Euploea core Cramer, Elymnias hypermenstra Linnaeus, Tirumala limiace Linnaeus, Danaus chryssipus Linnaeus and $D$. genutia Cramer. Hesperiidae and Lycaenidae families remained devoid of any frequently observed species. Tiger butterflies (Danaus genutia Cramer, D. chrysippus Linnaeus, D. melanippus Cramer and Tirumala limniace Cramer) (Image 4) were found to be unique to the mangroves. Except for the White Tiger, D. melanippus, the other three species were found to be more widely distributed in the Sundarbans. Moreover, by virtue of the presence of mangrove-associated plant species belonging to the Asclepiadaceae family: viz., Hoya R. $\mathrm{Br}$. and Tylophora R. Br. (Banerjee et al. 2002) that are preferred by the Striped Tiger, $D$. genutia as their larval 
food plants, the latter has been found to be widely frequent in the mangrove forests of the Sundarbans as well.

In the butterfly-poor Sundarbans deltaic region of eastern India, six of the recorded 76 species, approximately $8 \%$ of the butterflies are legally protected under the Indian Wildlife (Protection) Act, 1972 (Anonymous 1997). Of these, two are Schedule I, three are Schedule II and one is a Schedule IV species; the former two gain extreme importance because of the highest level of legal protection they receive in the country. Table 3 shows the taxonomic breakdown of these scheduled butterflies. Among the scheduled species, the Spotted Black Crow E. crameri has been recorded only from the deltaic regions of the Sundarbans and the mangrove and secondary forested regions of Orissa (Kehimkar 2008). Moreover, legally protected species like Gram Blue Euchrysops cnejus and Pea Blue Lampides boeticus are considered 'pests' for a variety of crops (NBAll 2013) and many-a-times eradicated in large numbers (mainly at their larval stages) along with other insect pests due to the indiscriminate use of pesticides in different agricultural tracts of the reclaimed areas of delta.

\section{CONCLUSION}

Habitat modifications and change in local climatic conditions (Danda et al. 2011), primarily due to human interventions, are the potent factors for organizing the butterfly community in the fragile ecosystems of Sundarbans. Such a change, as initiated in the otherwise lepidoptera-poor mangrove ecosystems of Sundarbans may interfere with their normal ecosystem functioning. Under the present scenario of increased stress towards these deltaic mangrove ecosystems, further studies on the exploration of larval food plants and niche specificities of the unique species (like the Tiger butterflies, Danaus genutia) in this region may help, in the long run, to consider them as focal species (viz., indicator species) for conservation programmes and ecosystem management of this highly threatened and bio diverse deltaic region.

\section{REFERENCES}

Agrawala, S., T. Ota, A.U. Ahmed, J. Smith \& M. van Aalst (2003). Development and Climate Change in Bangladesh: Focus on Coastal Flooding and the Sundarbans. Report no. COM/ENV/EPOC/DCD/ DAC(2003)3/FINAL. Organisation for Economic Cooperation and
Development, Paris, 70pp.

Anonymous (1997). The Wildlife (Protection) Act, 1972 (as amended upto 1993) with rules uptil 1995. Natraj Publishers, Dehra Dun, 158pp.

Banerjee L.K., T.A. Rao, A.R.K. Shastry \& D. Ghosh (2002). Diversity of Coastal Plant Communities in India. Kolkata: ENVIS-EMCBTAP, Botanical Survey of India, Ministry of Environment and Forests, 524pp.

Chaudhuri, A.B. \& A. Choudhury (1994). Mangroves of the Sundarbans. Volume 1: India. World Conservation Union, Gland, 247pp.

Chowdhury S. (2011). Ecology of Fiddler Crabs in the Sundarban Mangrove Ecosystems, West Bengal, India. PhD Thesis. School of Oceanographic Studies, Jadavpur University, 153pp.

Chowdhury, S. \& R. Soren (2009). Rediscovery of Small Salmon Arab, Colotis amata Fabricius from Saline and Semi-Saline Areas of West Bengal, India. Journal of Threatened Taxa 1(6): 351-352; http:// dx.doi.org/10.11609/JoTT.02127.351-2

Colwell, R.K. (2013). EstimateS: Statistical Estimation of Species Richness and Shared Species from Sample. Version 9.1.0. Freeware for Windows and Mac OS (Internet). <http://viceroy.eeb.uconn.edu/ EstimateS>. Downloaded on 8 July 2013.

Corbet, A.S. \& H.M. Pendlebury (1992). The Butterflies of Malay Peninsula, $4^{\text {th }}$ edition (Revised by J.N. Eliot). Malaysian Nature Society, Kuala Lampur, 594pp.

Danda, A.A., G. Sriskanthan, A. Ghosh, J. Bandyopadhyay \& S. Hazra (2011). Indian Sundarbans Delta - A Vision. World Wide Fund for Nature-India, New Delhi, 40pp.

Darwin, C. (1859). The Origin of Species by Means of Natural Selection. John Murray, London, 502pp.

Elton, C. (1946). Competition and the structure of ecological communities. Journal of Animal Ecology 15: 54-68; http://dx.doi. org/10.2307/1625

Evans, W.H. (1932). The Identification of Indian Butterflies. Bombay Natural History Society, Bombay, 464pp.

Fruhstorfer, H. (1910). Familie Danaidae [part], pp. 191-200, 209-216, 225-272. In: Seitz, A. (ed.). Die Gross-Schmetterlinge de Erde. Band IX. Die Tagfalter. A. Kernen, Stuttgart, 1197pp.

Gotelli, N.J. \& R.K. Colwell (2001). Quantifying Biodiversity: procedures and pitfalls in the measurement and comparison of species richness. Ecology Letters 4: 379-391; http://dx.doi.org/10.1046/j.14610248.2001.00230.x

Huq, S., Z. Karim, M. Asaduzzaman \& F. Mahtab (eds.) (1999). Vulnerability and Adaptation to Climate Change for Bangladesh. Kluwer Academic Publishers, Dordrecht, The Netherlands, 147pp.

IUCN (2011). The IUCN Red List of Threatened Species. Version 2011.2 <http://www.iucnredlist.org>. On-line version dated 15 April 2012.

Järvinen, O. (1982). Species-to-genus ratios in biogeography: a historical note. Journal of Biogeography 9: 363-370; http://dx.doi. org $/ 10.2307 / 2844723$

Kathiresan, K., \& B.L. Bingham (2001). Biology of mangroves and mangrove ecosystems. Advances in Marine Biology 40: 81-251.

Kehimkar, I. (2008). Book of Indian Butterflies. Bombay Natural History Society, Oxford University Press, Mumbai and Delhi, 513pp.

Magurran, A.E. (2004). Measuring Biological Diversity. Blackwell Science Ltd., UK, 256pp.

Mandal, A.K. \& N.C. Nandi (1989). Fauna of Sundarban Mangrove Ecosystem, West Bengal, India. Fauna of Conservation Areas: 3. Zoological Survey of India, Kolkata, 116pp.

Mandal, D.K. \& D.R. Maulik (1997). Insecta: Lepidoptera: Rhopalocera Papilionidae and Danaine Nymphalidae, pp. 755-793. In: Director, Zoological Survey of India (ed.). State Fauna Series 3: Fauna of West Bengal. Part 7. Zoological Survey of India, Kolkata, 793pp.

Naskar, K.R. \& R.N. Mandal (1999). Ecology and Biodiversity of Indian Mangroves. Daya Publishing House, Delhi, 754pp.

NBAll (2013). Castalius rosimon, Euchrysops cnejus and Lampides boeticus. In: Insects in Indian Agroecosystems. 2013 National Bureau of Agriculturally Important Insects. <http://www.nbaii.res.in/ insectpests/index.php>. Downloaded on 02 September 2013.

New, T.R. (2011). Launching and steering flagship Lepidoptera for conservation benefit. Journal of Threatened Taxa 3(6): 1805-1817; 
http://dx.doi.org/10.11609/JoTT.o2621.1805-17

Sanyal, P., L.K. Banerjee \& M.K. Choudhury (1984). Dancing Mangals of Indian Sundarbans. Journal of the Indian Society for Coastal Agricultural Research 2(1): 10-16.

Severiano, J.S, A.N. Moura, H.S.B. Oliviera, M.K. Cordeiro-Araujo \& E.W. Dantas (2012). Micro-phytoplankton richness in Contas River, state of Bahia, northeastern Brazil. Check List 8(2): 218-223.

Simberloff, D. (1978). Use of Rarefaction and Related Methods in Ecology, pp. 150-165. In: Dickson, K.L., J. Jr. Cairns \& R.J. Livingston (eds.). Biological Data in Water pollution Assessment: Quantitative and Statistical Analyses. American Society for Testing and Materials, Philadelphia, PA, USA.

Talbot, G. (1949). The fauna of British India including Ceylon and Burma - Butterflies 2. Taylor and Francis Ltd., London, 506pp.

van Nieukerken E.J., L. Kaila, I.J. Kitching, N.P. Kirstensen, D.C. Lees, J. Minet, C. Mitter, M. Mutanen, J.C. Regier, T.J. Simonsen, N. Wahlberg, S.-H. Yen, R. Zahiri, D. Adamski, J. Baixeras, D. Bartsch, B.A. Bengtsson, J.W. Brown, S.R. Bucheli, D.R. Davis, J. D. Prins, W.D.
Prins, M.E. Epstein, P. Gentili-Poole, C. Gielis, P. Hättenschwiler, A. Hausmann, J.D. Holloway, A. Kallies, O. Karsholt, A. Y. Kawahara, S. Koster, M.V. Kozlov, J.D. Lafontaine, G. Lamas, J.F. Landry, S. Lee, M. Nuss, K.-T. Park, C. Penz, J. Rota, A. Schintlmeister, B.C. Schimdt, J.-C. Sohn, M.A. Solis, G.M. Termann, A.D. Warren, S. Weller, R.V. Yakovlev, V.V. Zolotuhin \& A. Zwick (2011). Order Lepidoptera Linnaeus, 1758. In: Zhang, Z.-Q. (ed.) Animal Biodiversity: An Outline of Higher-Level Classification and Survey of Taxonomic Richness. Zootaxa 3148: 212-221.

Whittaker, R.H. (1960). Vegetation of the Siskiyou Mountains, Oregon and California. Ecological Monographs 30: 279-338. http://dx.doi. org/10.2307/1943563

Wynter-Blyth, M.A. (1957). Butterflies of the Indian Region. Bombay Natural History Society, Bombay, 523pp.

Zöckler, C., S. Balachandran, G.C. Bunting, M. Fanck, M. Kashiwagi, E.G. Lappo, E.G. Maheswaran, A. Sharma, E.E. Syroechkovski \& K. Webb (2005). The Indian Sunderbans: an important wintering site for Siberian waders. Wader Study Group Bulletin 108: 42-46. 